\title{
Estudio de la cinética de precipitación inducida mediante la determinación de los diagramas Precipitación-Tiempo- Temperatura (PTT) en aceros microaleados $^{(\bullet)}$
}

\begin{abstract}
S.F. Medina ${ }^{(*)}$
Resumen Se describe un método desarrollado en el CENIM que permite estudiar la cinética de la precipitación inducida por la deformación en aceros microaleados. Mediante ensayos de torsión, se ha determinado la fracción recristalizada estáticamente de tres aceros microaleados con niobio, vanadio y titanio, respectivamente, a diferentes temperaturas y deformaciones. Cuando la precipitación comienza, la fracción recristalizada se desvía de la ecuación de Avrami y esto permite conocer el instante en el que la precipitación comienza $\left(P_{\mathrm{s}}\right)$ y el instante en que termina $\left(P_{\mathrm{f}}\right)$. De este modo, se pueden trazar las curvas de Precipitación-Tiempo-Temperatura (PTT) que muestran gráficamente la cinética de precipitación.

Palabras clave: Recristalización estática. Precipitación inducida. Diagramas PTT.

\section{Study of induced precipitation kinetics by means of Precipitation-Time-Temperature diagrams (PTT) determi- nation in microalloyed steels}

\begin{abstract}
A method developed in the CENIM is described which allows to study the induced precipitation kinetics by the deformation in microalloyed steels. By means of torsion tests the statically recristallized fraction of three microalloyed steels with $\mathrm{Nb}, \mathrm{V}$ or $\mathrm{Ti}$, has been determined to different temperatures and deformations. When the precipitation begins, the recrystallized fraction is separated of the Avrami's equation and this allows to know the instant the precipitation begins $\left(P_{\mathrm{s}}\right)$ and the instant the precipitation finishes $\left(P_{\mathrm{f}}\right)$. Thereby can be traced the Precipitation-Time-Temperature (PTT) diagrams which graphically show the precipitation kinetics.
\end{abstract}

Keywords: Static recrystallization. Induced precipitation kinetics. PTT diagrams.

\section{INTRODUCCIÓN}

Los aceros microaleados al niobio son los más utilizados, ya que los precipitados inducidos por la deformación son generalmente más pequeños que en los aceros microaleados con vanadio y titanio, mejorando así el límite elástico y la resistencia (1). Por otra parte, la temperatura crítica de recristalización

\footnotetext{
(•) Trabajo recibido el día 11 de abril de 1995

(*) Centro Nacional de Investigaciones Metalúrgicas (CSIC). Avda. de Gregorio del Amo, 8. 28040-Madrid (España).
}

estática (SRCT), o temperatura a la cual comienza la inhibición de la recristalización como consecuencia de la precipitación inducida, es más alta en los aceros microaleados al niobio para igual contenido de microaleante (2). En aceros con bajos contenidos de carbono $(<0,15 \%)$ y de niobio (del orden del 0,040 $\%)$ y aproximadamente 80 p.p.m. de nitrógeno, la SRCT es mayor de $1.000^{\circ} \mathrm{C}$ y, en consecuencia, el intervalo de temperaturas (SRCT- $A r_{3}$ ) es suficientemente amplio, y la aplicación de una o más deformaciones permitiría obtener un fuerte endurecimiento de la austenita (pancaking) y, consiguientemente, un grano ferrítico muy pequeño después de la transformación austenita $\rightarrow$ ferrita (3-7). 
Recientemente, se ha desarrollado un método para determinar simultáneamente la cinética de la recristalización estática, a temperaturas superiores e inferiores a la SRCT, y la cinética de la precipitación inducida por la deformación expresada por los diagramas Precipitación-Tiempo-Temperatura (PTT) (2 y 8-11).

Los diagramas PTT de aceros microaleados determinados por otros autores se realizaron mediante ecuaciones teóricas (12-15), por microscopía de transmisión (16) o con técnicas de relajación (17).

En este trabajo se estudia, mediante ensayos de torsión, la recristalización estática de tres aceros microaleados con diferente tipo de microaleante (niobio, titanio y vanadio) y se determinan los diagramas PTT de cada uno.

\section{PROCEDIMIENTO EXPERIMENTAL}

Los aceros estudiados se fabricaron por refusión bajo electroescoria (proceso ESR) en una instalación capaz de producir lingotes de $30 \mathrm{~kg}$. Sus composiciones se muestran en la tabla I y, como puede observarse, los contenidos de microaleantes son muy similares, lo que permitirá establecer consideraciones específicas sobre el comportamiento de los mismos. Los ensayos de torsión en caliente se realizaron en una máquina SETARAM, completamente automatizada y de gran precisión, cuya unidad de tiempo programable a efectos de cuantificar la fracción recristalizada fue de $0,001 \mathrm{~s}$.

Las probetas de torsión, de $50 \mathrm{~mm}$ de longitud útil y $6 \mathrm{~mm}$ de diámetro, fueron austenizadas a $1.230{ }^{\circ} \mathrm{C}$ durante $10 \mathrm{~min}$ y enfriadas rápidamente hasta la temperatura de ensayo. Es necesario señalar que a la temperatura de $1.230{ }^{\circ} \mathrm{C}$ los precipitados de niobio y vanadio se disolvieron completamente, pero no ocurrió así con los nitruros de titanio, que sólo se disolvieron parcialmente.
Para asegurar que los ensayos se realizaban en fase austenítica, se determinaron las temperaturas $\mathrm{Ar}_{3}$ a una velocidad de enfriamiento de $0,2{ }^{\circ} \mathrm{C} / \mathrm{s}$ (Tabla I). Las deformaciones equivalentes aplicadas fueron de 0,20 y 0,35 , y la velocidad de deformación fue de $3,63 \mathrm{~s}^{-1}$, calculadas sobre la superficie cilíndrica de la probeta, de acuerdo con el criterio de plasticidad de Von Mises (18).

\section{RESULTADOS Y DISCUSIÓN}

Primeramente, se midió el tamaño del grano austenítico a la temperatura de austenización (1.230 ${ }^{\circ} \mathrm{C}$ durante $10 \mathrm{~min}$ ) de los tres aceros mediante el temple y posterior análisis metalográfico de 20 campos aplicando la técnica de la intersección lineal. Los resultados se muestran en la tabla I, donde puede apreciarse que el acero al titanio muestra el menor tamaño de grano, como era de esperar, puesto que es conocido el efecto inhibidor del titanio debido a la presencia de los precipitados no disueltos.

La fracción recristalizada se determinó aplicando el método conocido como back extrapolation (19). La figura 1 muestra la fracción recristalizada frente al tiempo para el acero al niobio, determinada para una deformación de 0,20 , velocidad de deformación de $3,63 \mathrm{~s}^{-1} \mathrm{y}$ a diferentes temperaturas. Se observa que comienza a formarse una meseta sobre la curva correspondiente a $1.025^{\circ} \mathrm{C}$ y, una vez que finaliza, la curva vuelve a ascender, completándose la recristalización. La formación de la meseta se debe a la precipitación inducida, y su comienzo y fin se identifican con el comienzo y el final de la precipitación inducida (2 y 8-11), lo que se tendrá en cuenta en la construcción de los diagramas PTT, como se verá más adelante. Las curvas que corresponden a temperaturas superiores a 1.025 ${ }^{\circ} \mathrm{C}$ muestran la forma habitual de la ley de Avrami para la recristalización. A temperaturas inferiores a

TABLA I.- Composición química, $\%$, temperatura crítica de transformación, $A r_{3}\left(0,2{ }^{\circ} \mathrm{C} / \mathrm{s}\right)$ y tamaño de grano austenítico, $D_{\gamma}$, a $1.230^{\circ} \mathrm{C}$ durante $10 \mathrm{~min}$, de los aceros estudiados

TABLE I.-Chemical composition, wt \%, transformation critical temperature, $\operatorname{Ar}_{3}\left(0,2{ }^{\circ} \mathrm{C} / \mathrm{s}\right)$ and austenite grain size, $\mu \mathrm{m}$, at $1.230^{\circ} \mathrm{C}$ during $10 \mathrm{~min}$ for the used steels

\begin{tabular}{|c|c|c|c|c|c|c|c|c|c|}
\hline $\begin{array}{c}\text { Acero } \\
\mathrm{al}\end{array}$ & $\mathrm{C}$ & $\mathrm{Si}$ & $\mathrm{Mn}$ & $\mathrm{Nb}$ & $\mathrm{V}$ & $\mathrm{Ti}$ & $\begin{array}{c}\mathrm{N}, \\
\text { p.p.m. }\end{array}$ & $\begin{array}{c}A r_{3}, \\
{ }^{\circ} \mathrm{C}\end{array}$ & $\begin{array}{c}D_{\gamma}, \\
\mu \mathrm{m}\end{array}$ \\
\hline $\mathrm{Nb}$ & 0,11 & 0,24 & 1,23 & 0,042 & - & - & 112 & 786 & 122 \\
\hline $\mathrm{V}$ & 0,11 & 0,24 & 1,10 & - & 0,043 & - & 105 & 784 & 172 \\
\hline $\mathrm{Ti}$ & 0,15 & 0,27 & 1,15 & - & - & 0,055 & 100 & 779 & 95 \\
\hline
\end{tabular}




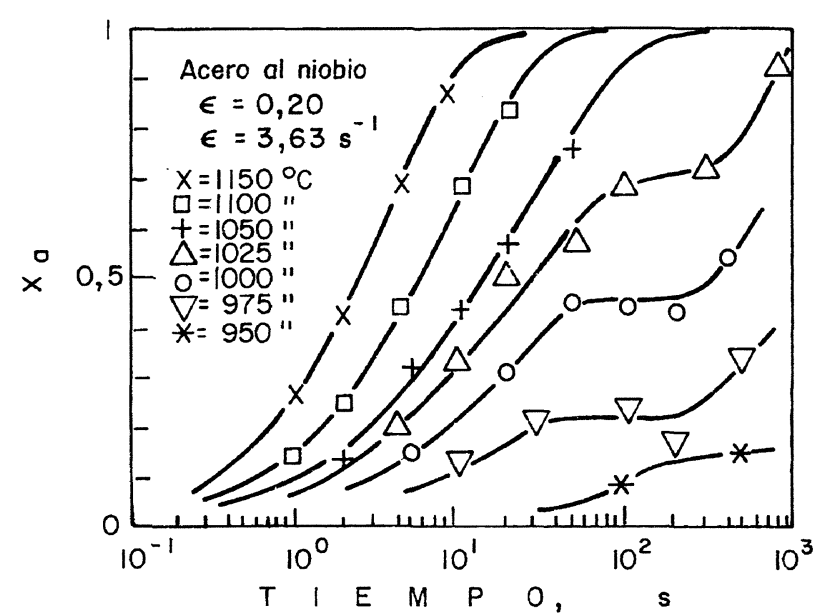

FIG. 1.- Fracción recristalizada, $X_{\mathrm{a}}$, frente al tiempo. Acero al niobio.

FIG. 1.-Recrystallized fraction, $\mathrm{X}_{\mathrm{a}}$, vs time. $\mathrm{Nb}$ steel.

$1.025{ }^{\circ} \mathrm{C}$, las curvas correspondientes muestran siempre la meseta, cuyo comienzo va teniendo lugar a fracciones recristalizadas cada vez menores.

La figura 2 ilustra la fracción recristalizada del acero al vanadio para la deformación de 0,20 y temperaturas de $1.100,1.000,900$ y $850^{\circ} \mathrm{C}$, respectivamente, observándose que la curvas correspondientes a 900 y $850{ }^{\circ} \mathrm{C}$ muestran una meseta originada por la formación de precipitados de vanadio que bloquean momentáneamente el progreso de la recristalización. La figura 3 presenta la fracción recristalizada para el acero al titanio, deformación de 0,20 y a diferentes temperaturas. Ahora, la meseta comienza a presentarse sobre la curva de $875^{\circ} \mathrm{C}$.

Las curvas correspondientes a la deformación de 0,35 presentaban formas similares a las anteriores.

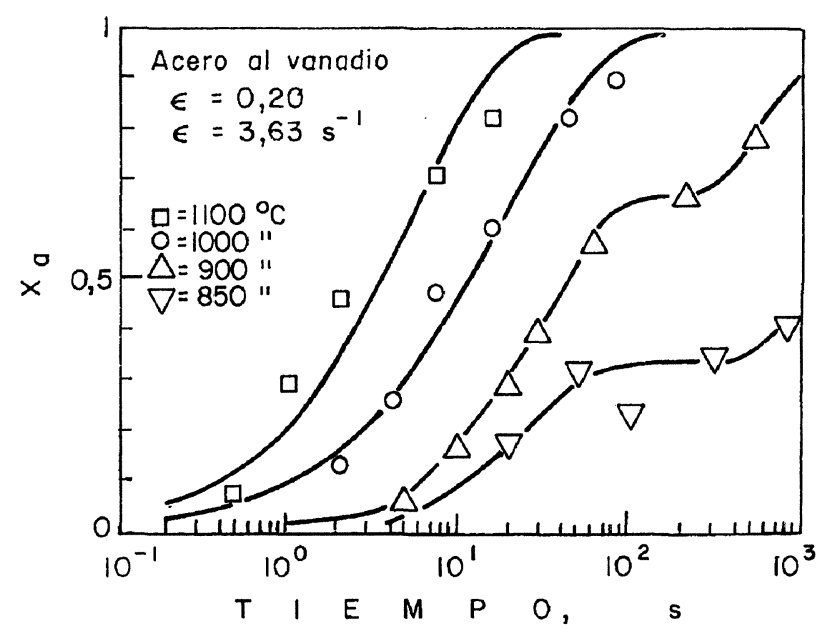

FIG. 2.- Fracción recristalizada, $X_{a}$, frente al tiempo. Acero al vanadio.

FIG. 2.-Recrystallized fraction, $\mathrm{X}_{\mathrm{a}}$, vs time. $V$ steel.

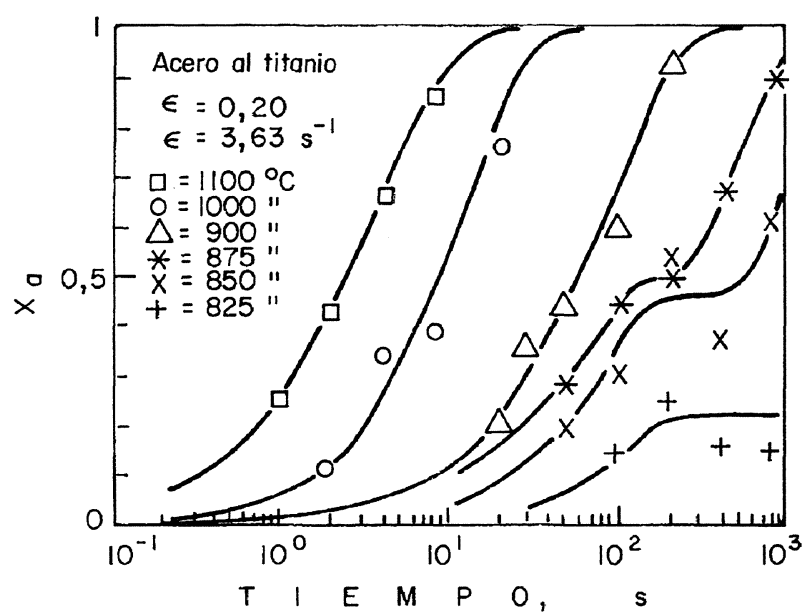

FIG. 3.- Fracción recristalizada, $X_{\mathrm{a}}$, frente al tiempo. Acero al titanio.

FIG. 3.- Recrystallized fraction, $\mathrm{X}_{\mathrm{a}}$, vs time. Ti steel.

A igual temperatura, en este caso la recristalización es más rápida; es decir, las curvas se desplazan hacia tiempos menores, apreciándose también una tendencia a que la aparición de la meseta se produzca a temperaturas ligeramente inferiores. De los resultados anteriores, se deduce que en todos los aceros microaleados, cualquiera que sea su naturaleza, se produce la precipitación inducida, y que ésta es capaz de bloquear momentáneamente la recristalización. Si esta inhibición momentánea se aprovecha en la laminación o en cualquier otro proceso de conformación en caliente, podría obtenerse una austenita muy deformada y, en consecuencia, una ferrita muy fina después de la transformación $\gamma \rightarrow \alpha$.

Una representación logarítmica del tiempo $\left(t_{0,5}\right)$ correspondiente a la fracción recristalizada de 0,5 , frente al inverso de la temperatura absoluta $(1 / T)$, permite determinar la energía de activación $(Q)$ para la recristalización estática. La figura 4 muestra un ejemplo, concretamente para el acero al niobio, donde se han representado los valores de $t_{0,5}$ para las dos deformaciones. La energía de activación viene dada por la pendiente de cada curva multiplicada por la constante universal de los gases $(R=$ $\left.8,318 \mathrm{Jmol}^{-1} \mathrm{~K}^{-1}\right)$, de acuerdo con el modelo aceptado por la mayoría de los investigadores para el parámetro $t_{0,5}: t_{0,5} \propto \exp (Q / R T)$. Antes de comenzar la precipitación inducida se observa que la energía de activación es constante, con un valor de $262.000 \mathrm{Jmol}^{-1}$ que corresponde al tramo lineal de la curva; por debajo de una cierta temperatura, anteriormente denominada SRCT, la energía de activación pasa a ser función de la temperatura, coincidiendo con el tramo no lineal. Del paralelismo de ambas rectas se deduce que la energía de activación es independiente de la deformación. 


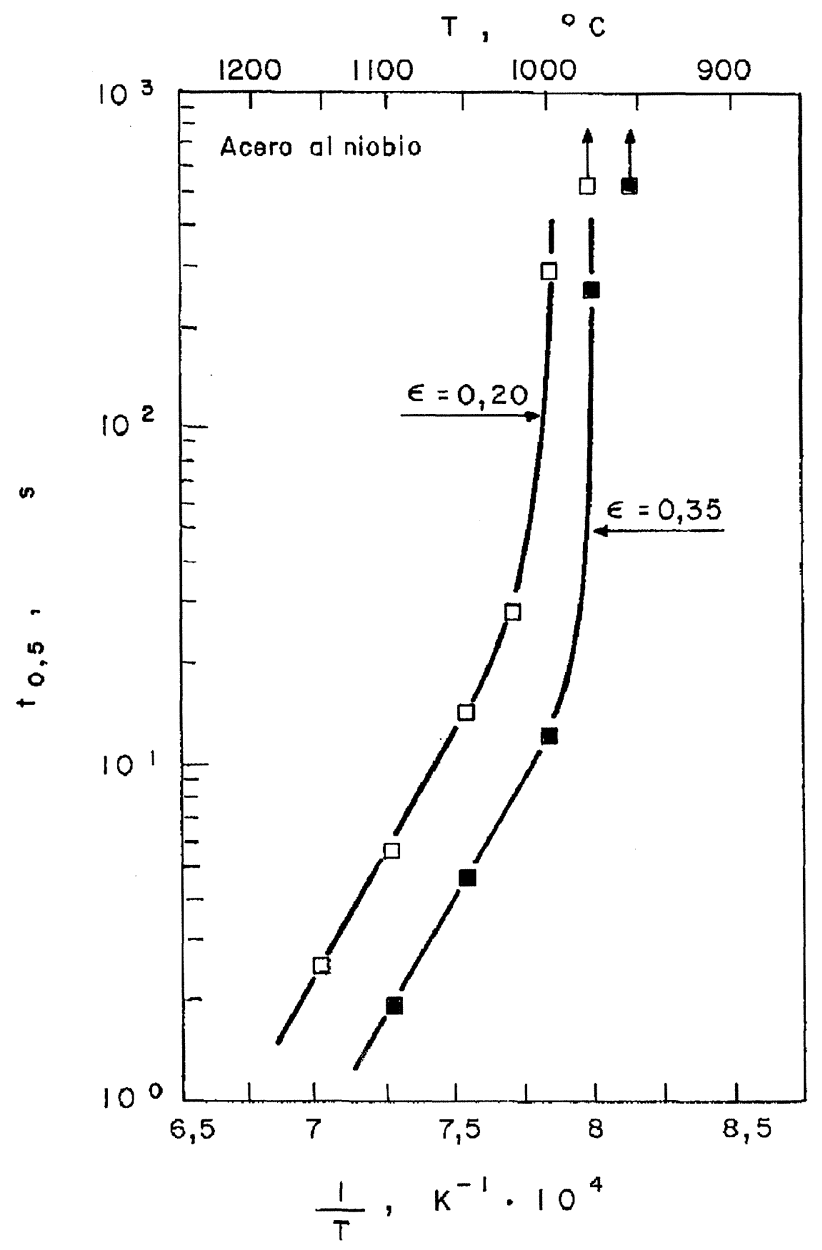

FIG. 4.- Parámetro $t_{0,5}$ frente al inverso de la temperatura. Acero al niobio.

FIG. 4.- Plot of $\mathrm{t}_{0.5}$ against the reciprocal of the temperature. $\mathrm{Nb}$ steel.

Una ulterior representación de $Q$, también frente a $1 / T$, permitirá calcular finalmente el valor de SRCT para cada acero, deformación y tamaño de grano austenítico. La figura 5 muestra un ejemplo, también para el acero al niobio, apreciándose que una mayor deformación hace descender el valor de SRCT, que viene dado por la intersección de la línea horizontal con la inclinada. De este modo, se determinó también la SRCT para los aceros al vanadio y al titanio, cuyos valores se muestran en la tabla II. Una comparación de los valores de SRCT con las temperaturas deducidas de los productos de solubilidad (20) permite deducir, a su vez, con buena aproximación la naturaleza química de los precipitados (Tabla II), responsables de la inhibición de la recristalización estática. Así, en el acero al niobio, los precipitados son carbonitruros de niobio, aunque posiblemente el carácter de nitruro sea preponderante. En el acero al vanadio son nitruros, ya que los carburos se formarían a temperaturas mucho más bajas. En el acero al titanio se hallan nitruros formados a temperaturas próximas a las de

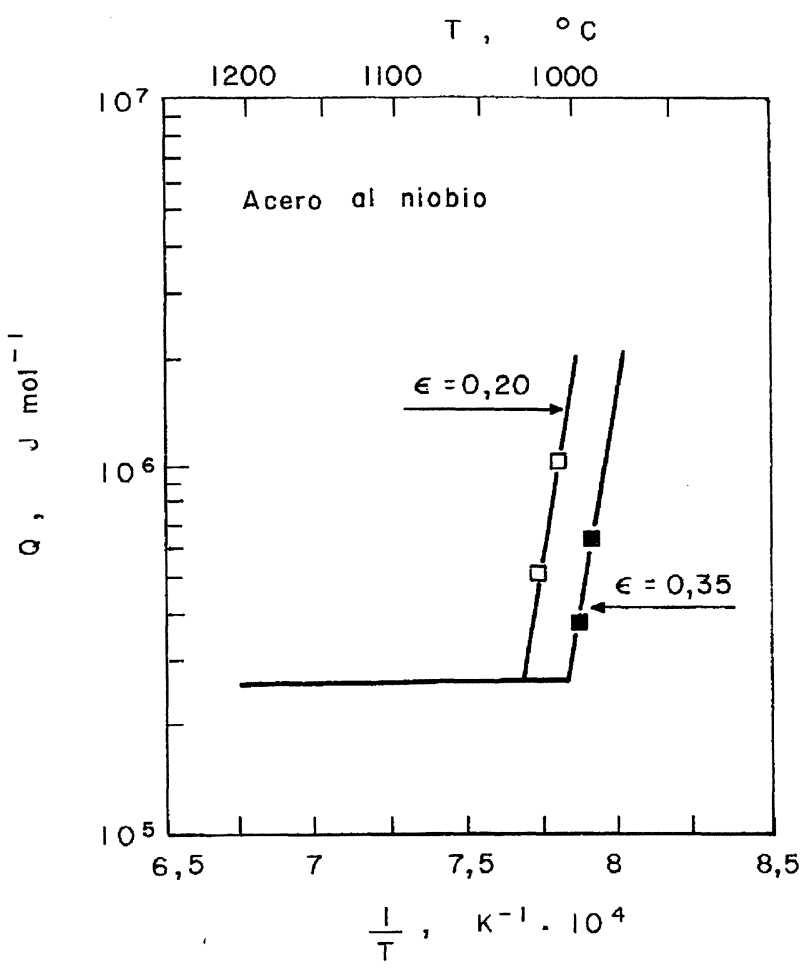

FIG. 5.- Energía de activación, $Q$, frente al inverso de la temperatura. Acero al niobio.

FIG. 5.- Plot of the activation, Q, against the reciprocal of the temperature. $\mathrm{Nb}$ steel.

solidificación, pero los precipitados inducidos por la deformación, responsables de la inhibición de la recristalización, son carburos. Esta última afirmación es obvia si se tiene en cuenta que el titanio en solución a $1.230{ }^{\circ} \mathrm{C}$ durante $10 \mathrm{~min}$ era de aproximadamente el 0,022 \% (21) y, por consiguiente, el titanio, precipitado a dicha temperatura, fue el

TABLA II.- Temperaturas críticas de recristalización, SRCT, y temperaturas de solubilidad, $T_{\mathrm{s}}$, de nitruros y carburos de los aceros estudiados

TABLE II.- Static recrystallization critical temperature, SRCT, and solubility temperature, $\mathrm{T}_{\mathrm{s}}$,

(nitrides and carbides) for the used steels

\begin{tabular}{|c|c|c|c|}
\hline $\begin{array}{c}\text { Acero } \\
\text { al }\end{array}$ & $\begin{array}{c}\text { SRCT, }{ }^{\circ} \mathrm{C} \\
\epsilon=0,20\end{array}$ & $\begin{array}{c}\text { SRCT, }{ }^{\circ} \mathrm{C} \\
\epsilon=0,35\end{array}$ & $\begin{array}{c}\text { Precipitado: } \\
T_{s},{ }^{\circ} \mathrm{C}\end{array}$ \\
\hline $\mathrm{Nb}$ & 1.030 & 1.005 & $\begin{array}{c}\mathrm{NbN}: 1.114 \\
\text { NbC: } 1.095\end{array}$ \\
\hline $\mathrm{V}$ & 903 & 880 & $\begin{array}{r}\text { VN: } 1.013 \\
\text { VC: } 799\end{array}$ \\
\hline $\mathrm{Ti}$ & 908 & 897 & $\begin{array}{l}\text { TiN: } 1.470^{*} \\
\text { TiC: } 1.068^{*}\end{array}$ \\
\hline
\end{tabular}

* Calculado con el titanio disuelto $(0,022 \%)$ a $1.230{ }^{\circ} \mathrm{C}$ durante $10 \mathrm{~min}(21)$. 
$0,033 \%$. La relación estequiométrica ( $\mathrm{Ti} / \mathrm{N}=3,4)$ de los nitruros de titanio (TiN), indica que prácticamente todo el nitrógeno se encontraba formalmente precipitado a la temperatura de austenización y, por tanto, el titanio en solución y posteriormente precipitado por acción de la deformación se halla en forma de carburos. Obviamente, en todos los casos, la SRCT siempre es menor que la temperatura de solubilidad, ya que la precipitación inducida por la deformación se aleja de las condiciones termodinámicas de equilibrio, con las que se determinaron los productos de solubilidad.

Los valores de la tabla II indican que, para el mismo contenido de microaleante, los aceros microaleados con niobio presentan la SRCT mayor, lo que se traduce en mayores facilidades para conseguir el pancaking de la austenita en el transcurso de la laminación.

De las curvas que presentan una meseta (Figs. 1, 2 y 3$)$, es posible deducir los tiempos iniciales $\left(P_{\mathrm{s}}\right)$ y finales $\left(P_{\mathrm{f}}\right)$ de la precipitación inducida y, por tanto, se podrán dibujar los diagramas PTT. La figura 6 muestra el diagrama PTT para el acero al niobio, observándose que el periodo mínimo de incubación, coincidente con la "nariz" de la curva, depende del contenido de niobio y de la deformación. Es necesario señalar que las curvas $P_{\mathrm{s}}$ y $P_{\mathrm{f}}$ tienen por asíntota la horizontal definida por SRCT, siendo de gran ayuda en el trazado de las mismas. Se observa que un incremento de la deformación disminuye el periodo de incubación, aunque es conocido que dicha influencia está condicionada por el contenido de niobio, siendo menor a medida que el contenido aumenta (9).

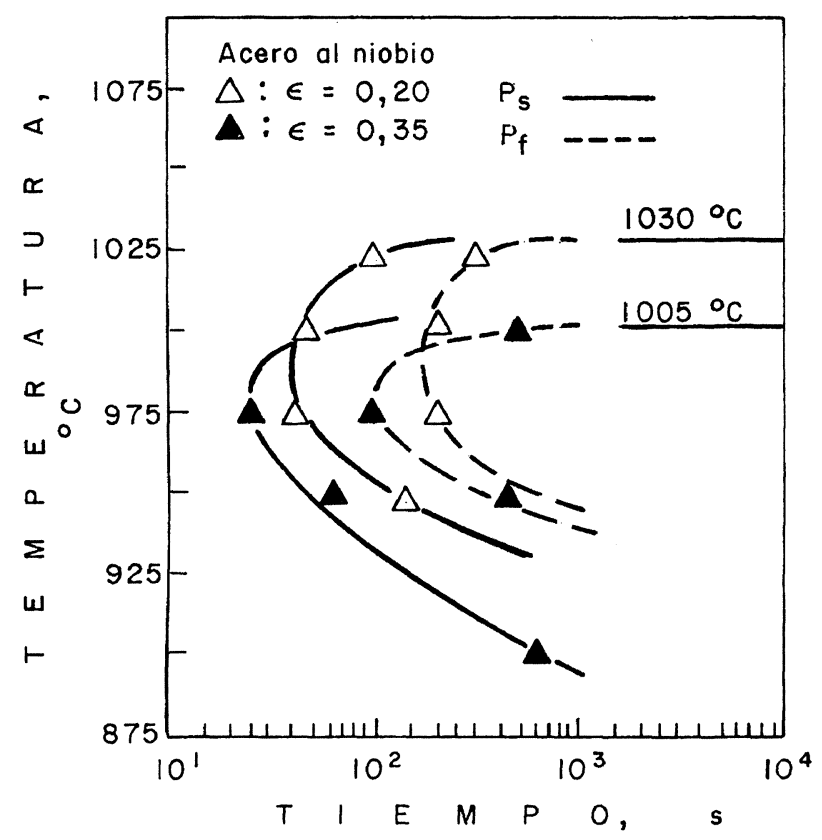

FIG. 6.- Diagramas PTT para el acero al niobio.

FIG. 6.-PTT diagrams for $\mathrm{Nb}$ steel.
La figura 7 muestra el diagrama PTT para el acero al vanadio. La diferencia sustancial con respecto al diagrama anterior (acero al niobio) es el valor más bajo de SRCT y, en consecuencia, la temperatura más baja de la "nariz" de la curva (NT). Finalmente, la figura 8 ilustra el diagrama PTT para el acero microaleado al titanio. La influencia del contenido de microaleante se ha estudiado (8-11), conociéndose que un aumento de dicho contenido tiende a disminuir el período de incubación de la precipitación.

\section{CONCLUSIONES}

- El método expuesto permite determinar los diagramas PTT y conocer, por tanto, la cinética de precipitación.

- A igual contenido de microaleante, los aceros al niobio presentan valores más altos de SRCT.

- A pesar del bajo porcentaje de titanio en solución a $1.230{ }^{\circ} \mathrm{C}$ durante $10 \mathrm{~min}$, del orden del $0,022 \%$, el acero al titanio presenta una SRCT próxima a la del acero al vanadio debido a la gran afinidad del titanio por el carbono.

- Pequeñas cantidades de microaleante son suficientes para formar precipitados.

- Un aumento de la deformación disminuye el período de incubación.

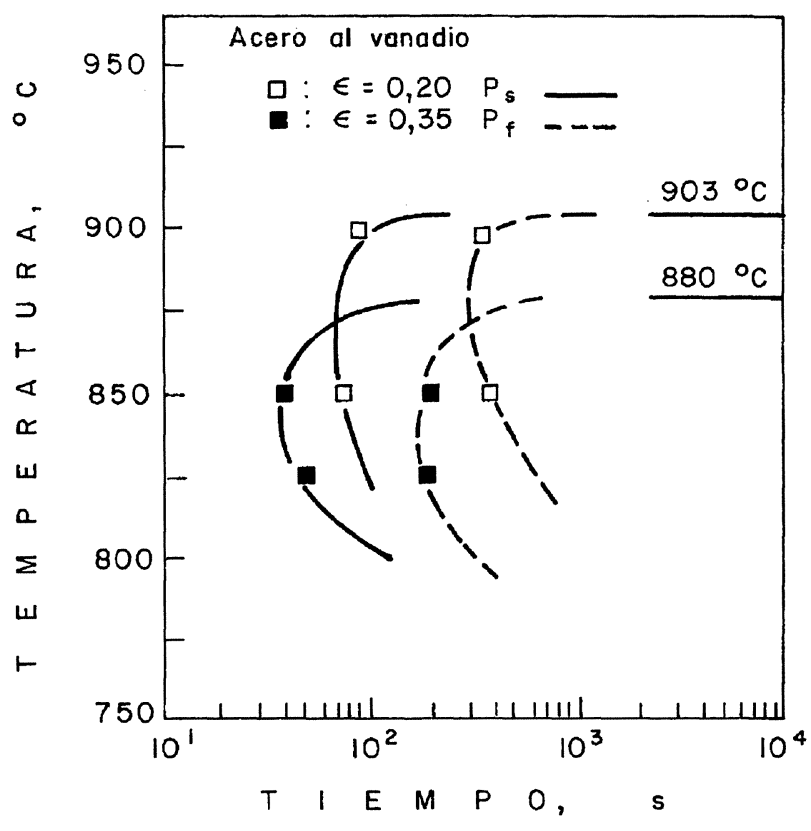

FIG. 7.- Diagramas PTT para el acero al vanadio.

FIG. 7.-PTT diagrams for $V$ steel. 


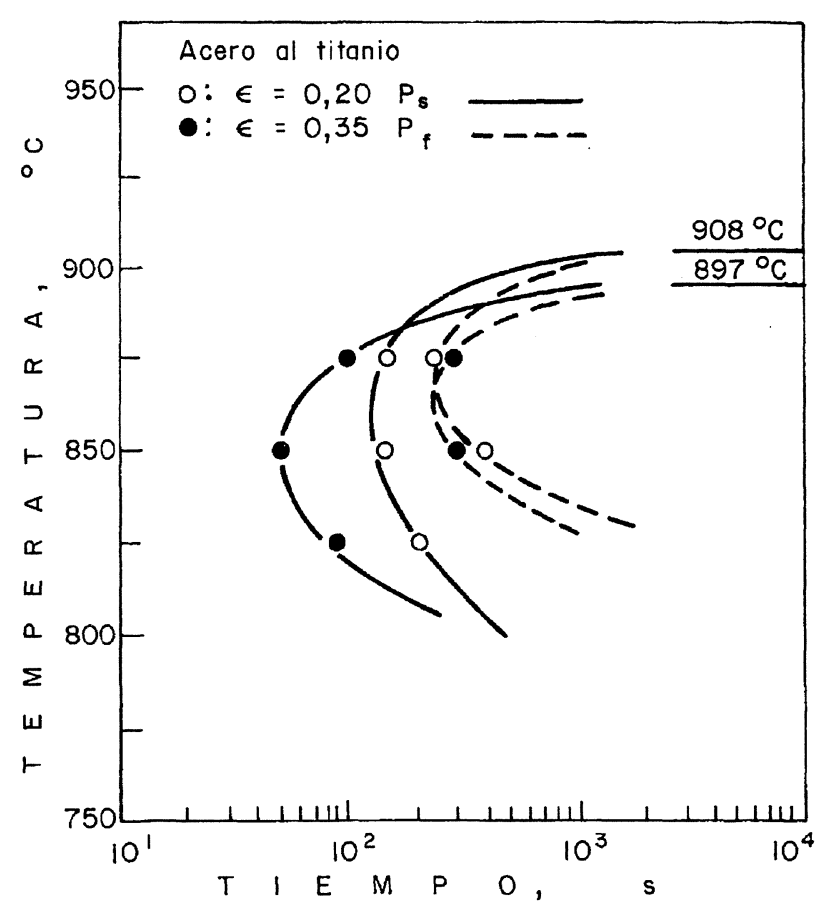

FIG. 8.- Diagramas PTT para el acero al titanio.

FIG. 8.-PTT diagrams for Ti steel.

\section{REFERENCIAS}

(1) Gladman, T. Ironmaking Steelmaking, 16 (4), 1989: 241245.

(2) Medina, S.F. y Mancilla, J.E. ISIJ Int., 33, (12), 1993: 1.257-1.264.

(3) Medina, S.F. y LóPEZ, V. ISIJ Int., 33 (5), 1992: 605-614.
(4) NAJAFI-ZADEH, A., Yue, S. y JonAS, J.J. ISIJ Int., 32 (2), 1992: 213-221.

(5) Pussegoda, L.N. y Jonas, J.J. ISIJ Int., 31 (3), 1991: 278-288.

(6) SAMuel, F.H., Yue, S., Jonas, J.J. y ZBinden, B.A. ISIJ Int., 29 (10), 1989: 878-886.

(7) Samuel, F.H., Yue, S., Jonas, J.J. y Barnes, K.R. ISIJ Int., 30 (3), 1990: 216-225.

(8) Medina, S.F. y Mancilla, J.E. Scr. Metall. Mater., 30 (1), 1994: 73-78.

(9) Medina, S.F. y Mancilla, J.E. Acta Metall. Mater., 42 (12), 1994: 3.945-3.951.

(10) Medina, S.F., Mancilla, J.E. y Hernández, C.A. ISIJ Int., 34 (8), 1994: 689-696.

(11) Medina, S.F. y Mancilla, J.E. Scr. Metall. Mater., 31 (3), 1994): 315-320.

(12) DutTA, B. y Sellars, C.M. Mater. Sci. Technol., 3 (3), 1987: 197-206.

(13) OKaguchi, S. y Hashimoto, T. ISIJ Int., 32 (3), 1992: 283-290.

(14) Park, S.H., Yue, S. y Jonas, J.J. Metall. Trans. A, 23A (6), 1992: 1.641-1.651.

(15) BaI, D.Q., Yue, S., Sun, W.P. y Jonas, J.J. Metall. Trans. A, 24A, (1993): 2.151-2.159.

(16) Le Bon, A., Rofes-Vernis, J. y Rossard, C. Met. Sci., 9, 1975: 36-40.

(17) Liu, W.J. y Jonas, J.J. Metall. Trans. A, 20A (8), 1989: 1.361-1.374.

(18) FAESSEL, A. Rev. Metall. CIT, 4, 1976: 875-892.

(19) ANDRAde, H.L., AKben, M.G. y Jonas, J.J. Metall. Trans. A, 14A (10), 1983: 1.967-1.977.

(20) NARITA, K. Trans. Iron Steel Inst. Jpn., 15, 1975: 145152.

(21) TuRKDOGAN, E.T. Iron Steelmaker, 16 (5), 1989: 61-75. 